resolution, two remain under the infertility team and one has ongoing abdominal pain. Median treatment duration was six months.

Conclusion This case series highlights the delay in diagnosis and the significant morbidity - particularly infertility - experienced by patients with genital TB. Samples were frequently not sent for culture. Raising awareness of TB within obstetrics and gynaecology and highlighting the importance of considering TB in patients from high incidence countries may help reduce diagnostic delay for these women.

\section{P185 IMPROVING THE ACCURACY OF MICROBIOLOGICAL DIAGNOSIS OF TB LYMPHADENITIS - IS A MULTIDISCIPLINARY APPROACH NECESSARY?}

A Saigal, HS Kalsi, R Sands, A Jayaratnam. Barking, Havering and Redbridge NHS Trust, Romford, UK

\subsection{6/thoraxjnl-2014-206260.314}

Introduction The gold standard for diagnosing tuberculosis (TB) is from culture of the organism from fluid or tissue. Histological analysis of surgical specimens is well-established, but microbiological analysis is less frequent. Our trust serves a population with a high incidence of TB. Therefore, patients who present with lymphadenopathy should always be considered for a diagnosis of TB and all specimens sent for microbiological and histological diagnosis.

Methods A retrospective analysis was undertaken of all patients diagnosed with TB lymphadenitis between 2009-2013 using the London TB Register (LTBR), case notes and laboratory data to identify the proportion diagnosed with microbiology data compared with histology data.

Results 324 patients were diagnosed with $\mathrm{TB}$ lymphadenitis from LTBR, of which 73\% (235/324) had lymph node (LN) specimens taken for microbiological or histological diagnosis.

233 patients had extrathoracic disease alone, of which $62 \%$ (144/233) had LN tissue sent for microbiology with 74\% yielding a positive culture. 75 patients had intrathoracic disease, of which $31 \%(23 / 75)$ had LN tissue sent for microbiology with $52 \%$ yielding a positive culture. In both groups, a greater percentage of LN tissue was sent for histo-cytological analysis than microbiology (see figure).

$75 \%(12 / 16)$ of patients with combined extrathoracic and intrathoracic disease had specimens sent for microbiology. 83\%

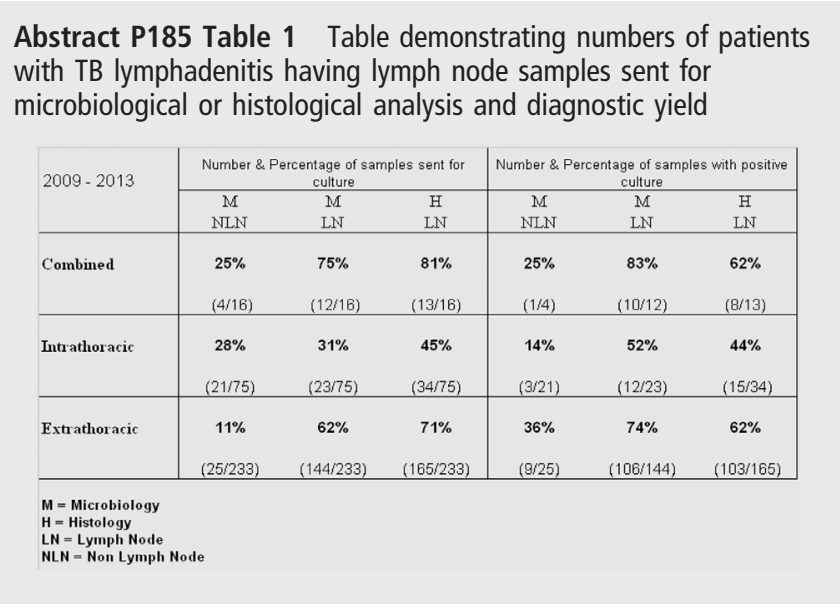

(11/16) gained a positive microbiological diagnosis from lymph node sampling.

Conclusion Microbiological specimens were more likely to be sent in patients with extrathoracic disease compared to those with intrathoracic disease. This may partly be explained by the fact that all intrathoracic lymph node sampling during this study period was undertaken at other centres, mostly through referrals from the lung cancer MDT. Therefore TB may not have been considered as a possible diagnosis.

However, a significant proportion of surgical samples taken locally did not have microbiology specimens sent, which potentially may have impacted on treatment outcomes.

This review highlights that more education should be undertaken locally with surgical and radiology departments and the lung MDT, emphasising the need for all lymph node specimens to be sent for both microbiological and histological analysis.

\section{P186 INTRATHORACIC LYMPH NODE TUBERCULOSIS - A COMPREHENSIVE CLINICAL DESCRIPTION}

${ }^{1} \mathrm{KJH}$ Kow, ${ }^{2} \mathrm{DW}$ Connell, ${ }^{2} \mathrm{~A}$ Singanayagam, ${ }^{2} \mathrm{D}$ Ap Dafydd, ${ }^{2} \mathrm{H}$ Jarvis, ${ }^{2} \mathrm{M}$ O'Donoghue, ${ }^{2} \mathrm{Ml}$ Wickremasinghe, ${ }^{2} \mathrm{~A}$ Lalvani, ${ }^{2} \mathrm{OM}$ Kon. ${ }^{1}$ Imperial College London, London, UK; ${ }^{2}$ St Mary's Hospital, Imperial College Healthcare NHS Trust, London, UK

\subsection{6/thoraxjnl-2014-206260.315}

Background Intrathoracic lymph node tuberculosis (ITLNTB) is an extra-pulmonary manifestation of tuberculosis (TB) and a predominant feature of primary TB in children. Historical literature supports the key role of lymph nodes in tuberculosis pathogenesis yet there is a paucity of literature describing ITLNTB in adults.

Methods This study comprehensively reviewed the clinical, radiological and pathological features of ITLNTB from 20092012 at a busy urban tuberculosis clinic.

Results 113 adult patients with ITLNTB were identified between 2009-2012. Patients were usually male, with a mean age of $41.5 \pm 15.8$ years and mostly from White, Black-African or Indian ethnic groups. $86 \%$ were non-UK born and most presented within 5-10 years of entering the country. $43 \%$ were asymptomatic. A subgroup of patients who were mycobacterial culture positive on endobronchial ultrasound sampling (EBUS) of intrathoracic lymph nodes were identified as patients with definite mycobacterial infection of the lymph nodes $(n=27)$.

Comparisons between symptomatic and asymptomatic groups in the whole cohort and EBUS culture positive subgroup demonstrated significant associations between symptoms and disease dissemination ( $\mathrm{p}=0.0002$ and $\mathrm{p}=0.01$ respectively); and symptoms and cytological response in the lymph nodes ( $p=0.02$ and $p=0.01$ respectively), suggesting the presence of a spectrum of disease reflected in congruent clinical and pathological responses (Table 1). Comparisons between disease sites affected also showed a significant association between host response in the lymph nodes and disease dissemination $(\mathrm{p}=0.006)$.

The presence of radiological necrosis, number of affected nodal stations, and largest node size were significantly greater in symptomatic patients in the whole cohort; with a similar trend observed in the EBUS culture positive subgroup.

In the EBUS culture positive subgroup, asymptomatic patients were identified significantly earlier following entry to the UK $(\mathrm{p}=0.01)$.

Discussion This study provides the first comprehensive clinical description of ITLNTB in adults. There is a spectrum of disease 
Abstract P186 Table 1 Comparison between symptomatic and asymptomatic groups from EBUS culture positive subcohort

\begin{tabular}{|c|c|c|c|c|}
\hline & & $\frac{\text { Symptomatic }}{(n=19)}$ & $\frac{\text { Asymptomatic }}{(\mathrm{n}=8)}$ & P-value \\
\hline \multicolumn{2}{|c|}{ Gender (M:F) } & $\begin{array}{l}68.4 \%: \\
31.6 \%\end{array}$ & $50 \%: 50 \%$ & 0.42 \\
\hline \multicolumn{2}{|c|}{ Age (mean $\pm S D$ ) (years) } & $35.58 \pm 12.81$ & $46.0 \pm 17.97$ & 0.12 \\
\hline \multirow{3}{*}{ Ethnicity } & White & $15.79 \%$ & $25 \%$ & \multirow{3}{*}{0.81} \\
\hline & Black-African & $26.32 \%$ & $37.5 \%$ & \\
\hline & Indian & $31.58 \%$ & $25 \%$ & \\
\hline \multirow{3}{*}{ Born in UK } & $\mathrm{Y}$ & $5.26 \%$ & $12.5 \%$ & \multirow{3}{*}{0.53} \\
\hline & $\mathrm{N}$ & $89.47 \%$ & $87.5 \%$ & \\
\hline & U & $5.26 \%$ & $0 \%$ & \\
\hline Duration in & $\mathrm{K}$ (median; IQR) & $12(4-19.5)$ & $2(1-6)$ & $0.01^{*}$ \\
\hline \multirow{5}{*}{ Exposures } & Diabetes & $0 \%$ & $14.29 \%$ & 0.33 \\
\hline & HIV & $5.26 \%$ & $0 \%$ & 0.75 \\
\hline & Travel endemic & $68.42 \%$ & $42.86 \%$ & 0.23 \\
\hline & Smoking & $13.33 \%$ & $14.29 \%$ & 1.00 \\
\hline & TB contact & $37.5 \%$ & $33.33 \%$ & 1.00 \\
\hline \multirow{5}{*}{$\begin{array}{l}\text { Disease } \\
\text { sites }\end{array}$} & Pulmonary & $47.37 \%$ & $0 \%$ & \multirow{5}{*}{$0.01^{*}$} \\
\hline & Pleural & $5.26 \%$ & $0 \%$ & \\
\hline & ETLN & $26.32 \%$ & $0 \%$ & \\
\hline & Skin & $0 \%$ & $12.5 \%$ & \\
\hline & Eye & $5.26 \%$ & $12.5 \%$ & \\
\hline \multicolumn{2}{|c|}{$\%$ Isolated } & $31.58 \%$ & $62.5 \%$ & \multirow{4}{*}{$0.03^{*}$} \\
\hline & $n+I T L N$ & $47.37 \%$ & $0 \%$ & \\
\hline \multicolumn{2}{|c|}{ \% EPTB (excl skin \& eye) } & $42.11 \%$ & $12.5 \%$ & \\
\hline \multicolumn{2}{|c|}{$\%$ Hypersens TB } & $5.26 \%$ & $25 \%$ & \\
\hline \multirow{2}{*}{ Cytology } & $\begin{array}{l}\text { Granulomatous } \\
\text { w/ caseation }\end{array}$ & $52.63 \%$ & $0 \%$ & \multirow[t]{2}{*}{$0.01^{*}$} \\
\hline & $\begin{array}{l}\text { Granulomatous } \\
\text { w/o caseation }\end{array}$ & $26.32 \%$ & $75 \%$ & \\
\hline
\end{tabular}

based on clinical severity, disease phenotype and diagnostic and radiological findings. Host response in the lymph nodes is reflected by both symptom manifestation and disease dissemination, implicating the lymph nodes in a critical role in the natural history of TB infection. Finally, a subclinical phenotype was identified, suggesting an early stage of disease progression in TB.

\section{P187 THE USE OF MOXIFLOXACIN FOR THE TREATMENT OF OPHTHALMIC TUBERCULOSIS}

${ }^{1} \mathrm{JL}$ Potter, ${ }^{2} \mathrm{R}$ Agrawal, ${ }^{1} \mathrm{C}$ Barraclough, ${ }^{2} \mathrm{~F}$ Rahman, ${ }^{1} \mathrm{H}$ Kunst, ${ }^{2} \mathrm{M}$ Westcott. ${ }^{1}$ Barts Health NHS Trust, London, UK; ${ }^{2}$ Moorfields Eye Hospital NHS Foundation Trust, London, UK

\subsection{6/thoraxjnl-2014-206260.316}

Background The number of patients we are treating for ophthalmic tuberculosis (TB) have increased year on year, from two in 2009 to twenty in 2013. A recent global review of the strategies used in the diagnosis and treatment of ophthalmic TB showed a wide disparity of diagnostic and treatment strategies. We present a review of our current practice and justification for out treatment regimens.

Methods We identified all the cases in our hospital treated for ophthalmic TB between 2009 and 2013. Age, gender, ophthalmic examination findings, blood tests, treatment regimens, including durations and outcomes, and adverse drug reactions were collected and analysed.

Results A total of 60 cases were identified. Mean age was 45.0 +/- 14.4 years. $61.7 \%$ were male. The most commonly used regimen was 2 months rifampicin, isoniazid, pyrazinamide and moxifloxacin followed by 10 months of rifampicin, isoniazid and moxifloxacin. A response to treatment, with no evidence of disease recurrence on cessation of therapy, was seen in $78.3 \%$ of cases. 5\% experienced hepatotoxicity requiring a change in treatment. There was no significant difference in either the success of treatment $(p=0.102)$ or the risk of hepatotoxicity $(p=0.264)$ between regimens with moxifloxacin $(n=43)$ or without it ( $n$ $=17) .32$ patients on moxifloxacin had ECGs of which 6
(18.8\%) newly developed a raised QTc. This resulted in moxifloxacin being stopped during the step-down phase of treatment in two patients. Maximum QTc was never found to be above 500 milliseconds and there were no episodes of documented arrhythmias or syncope.

Conclusions We recommend a treatment regimen including moxifloxacin in place of ethambutol so that any reported visual change is unlikely to be related to treatment, and we propose continuing moxifloxacin beyond the intensive phase, if tolerated, when culture is unavailable. We treat ophthalmic TB for the same duration as central nervous system TB. Our data shows that this is a safe and effective regimen but more evidence is required before recommending definitive guidelines.

\section{P188 INCREASED PULMONARY M. AVIUM-INTRACELLULARE ISOLATES ACCOUNT FOR MUCH OF THE NATIONAL RISE IN NON-TUBERCULOUS MYCOBACTERIA INCIDENCE, 2007-2012}

${ }^{1} \mathrm{NM}$ Shah, ${ }^{1} \mathrm{~J}$ Davidson, ${ }^{1} \mathrm{~L}$ Anderson, ${ }^{1} \mathrm{HL}$ Thomas, ${ }^{2} \mathrm{M}$ Lipman, ${ }^{1} \mathrm{I}$ Abubakar. ${ }^{1}$ Public Health England, London, UK; ${ }^{2}$ Royal Free London NHS Foundation Trust, London, UK

\subsection{6/thoraxjnl-2014-206260.317}

Introduction The incidence of nontuberculous mycobacteria (NTM) isolation from humans is increasing worldwide. In England, Wales and Northern Ireland (EW and NI) the reported rate of NTM more than doubled between 1996 and 2006. It is unclear if this trend has continued. We present an updated analysis with national NTM data from 2007 to 2012.

Methods All individuals with culture positive NTM isolates between 2007-2012 reported to Public Health England by the five mycobacterial reference laboratories serving EW and NI, were included. The annual incidence of NTM was calculated based on the year of the first positive NTM isolate from each individual.

Results 21,024 individuals had NTM culture positive samples. Over the study period the incidence rose from $5.57(\mathrm{n}=3126)$ to $7.63(\mathrm{n}=4454)$ per 100,000 population. The majority were male $(57 \%)$ and older $(71 \%>50$ years of age). $77 \%$ of individuals had a pulmonary isolate - and here the incidence increased from 3.97 to 6.05 per 100,000 population between 2007 and 2012. In those with extra-pulmonary samples it remained stable at 1.61 per 100,000 population. In patients

Table 1 indicates the seven most frequently reported organisms; M. avium-intracellulare (MAI) accounted for 35\% of isolates $(75 \%$ in people $>50$ years). $78 \%$ of these were from pulmonary samples. $42 \%$ of $\mathrm{M}$. abscessus isolates were in patients 60 years.

\begin{tabular}{llll}
$\begin{array}{l}\text { Abstract P188 Table } 1 \\
\text { samples 2007-2012 }\end{array}$ & Most common NTM reported from total \\
\hline & $\begin{array}{l}\text { Total number } \\
\text { of samples }\end{array}$ & $\begin{array}{l}\text { 2007 incidence } \\
\text { (per 100,000 }\end{array}$ & $\begin{array}{l}\text { 2012 incidence } \\
\text { (per 100,000 } \\
\text { population) }\end{array}$ \\
Organism & $\mathbf{2 0 0 7 - 1 2}$ & 1.90 & 2.80 \\
\hline M. avium-intracellulare & 7400 & 0.74 & 1.38 \\
M. gordonae & 3373 & 0.60 & 0.9 \\
M. chelonae & 2318 & 0.47 & 0.58 \\
M. fortuitum & 1681 & 0.42 & 0.29 \\
M. kansasii & 1299 & 0.34 & 0.34 \\
M. xenopi & 1065 & 0.26 & 0.38 \\
M. abscessus & 986 & & \\
\hline
\end{tabular}

